Supporting Information

\title{
Allosteric Communication with the Retinal Chromophore upon Ion Binding in a Light- Driven Sodium Ion-Pumping Rhodopsin
}

Akihiro Otomo, ${ }^{+}$Misao Mizuno,${ }^{+}$Keiichi Inoue,${ }^{\ddagger}$ Hideki Kandori, ${ }^{\mathbb{}}{ }^{\S} \S$ Yasuhisa Mizutani ${ }^{+*}$

+ Department of Chemistry, Graduate School of Science, Osaka University, 1-1 Machikaneyama, Toyonaka, Osaka 560-0043, Japan

‡ The Institute for Solid State Physics, The University of Tokyo, Kashiwa 277-8581, Japan

^ Department of Life Science and Applied Chemistry, Nagoya Institute of Technology, Showaku, Nagoya 466-8555, Japan

§ OptoBioTechnology Research Center, Nagoya Institute of Technology, Showa-Ku, Nagoya 466-8555, Japan 


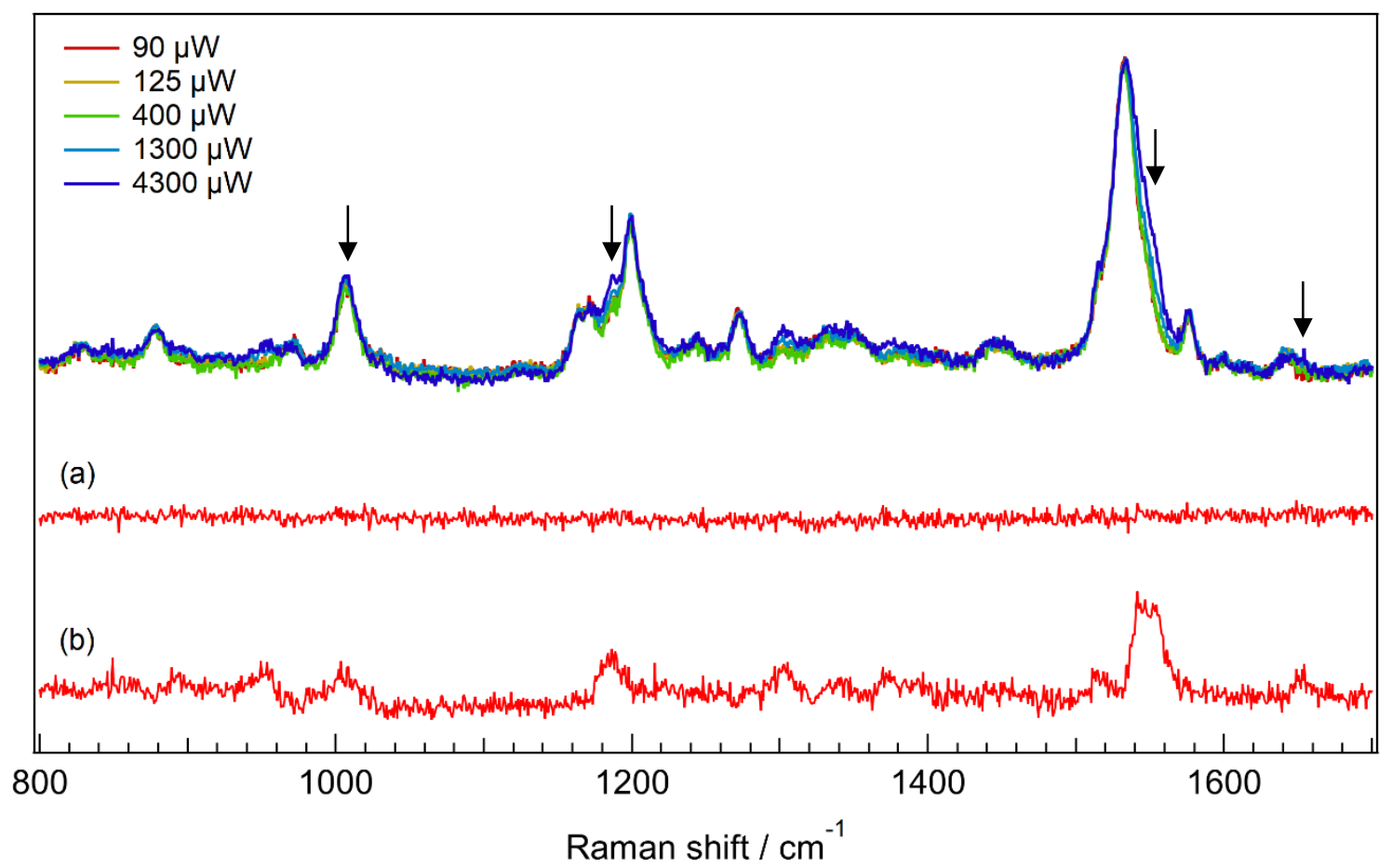

Figure S1. Probe power dependence of the resonance Raman spectra of nanodisc-reconstituted $\mathrm{KR} 2$ in the presence of $100 \mathrm{mM} \mathrm{Na}{ }^{+}$. Top traces show the spectra measured using probe powers of $90,125,400,1300$, and $4300 \mu \mathrm{W}$. The rotation speed of the spinning cell was set to $750 \mathrm{rpm}$. When the probe power is high, the contribution of the photointermediates generated in the laser irradiation volume appears in the spectra as shown by the black arrows. Trace a shows the difference spectrum between the $90-\mu \mathrm{W}$ spectrum and the $125-\mu \mathrm{W}$ spectrum. Trace b represents the difference spectrum between the $90-\mu \mathrm{W}$ spectrum and the $4300-\mu \mathrm{W}$ spectrum. Since there are no bands in trace a, a probe power of $125 \mu \mathrm{W}$ is sufficiently weak to prevent contamination of the spectra by undesirable photoproducts. By contrast, there are some RR bands due to the photointermediates in trace $b$. 

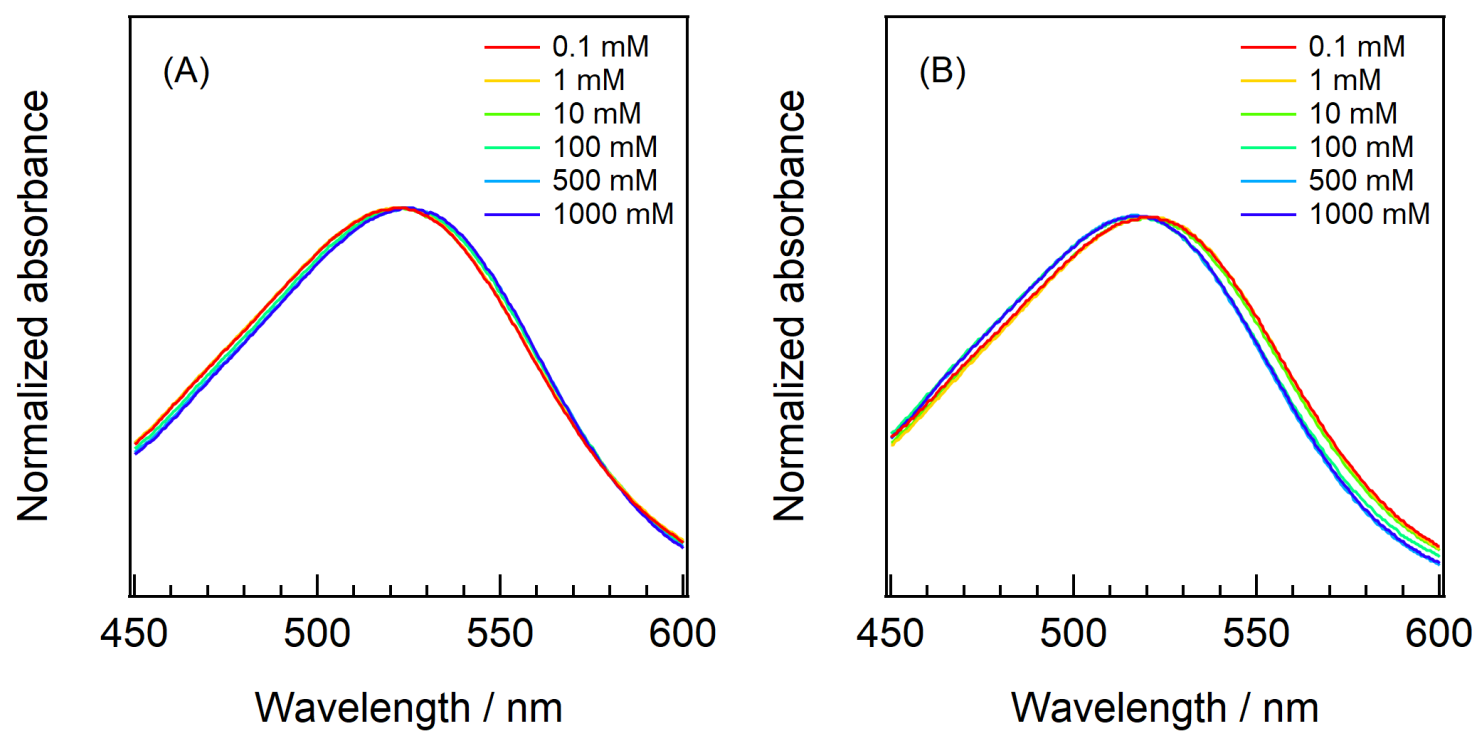

Figure S2. Absorption spectra of nanodisc-reconstituted KR2 over a range from 450 to $600 \mathrm{~nm}$ in the presence of $\mathrm{Na}^{+}(\mathrm{A})$ and $\mathrm{K}^{+}(\mathrm{B})$. Each cation concentration was adjusted by dialysis. All spectra are normalized at the absorption maximum wavelength. Concentration increase of $\mathrm{Na}^{+}$and $\mathrm{K}^{+}$ resulted in the redshift and the blueshift of the absorption maximum wavelength, respectively. 

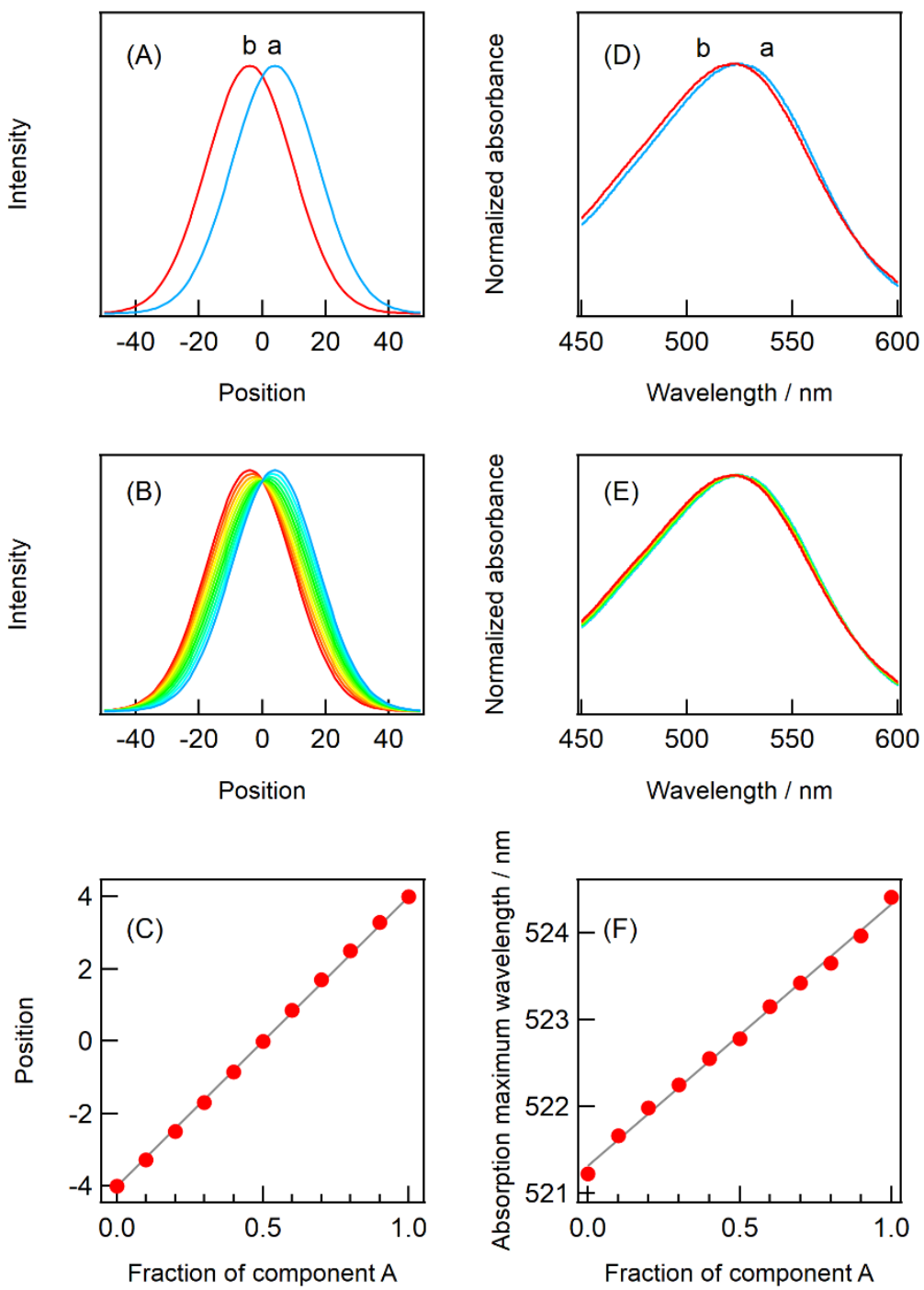

Figure S3. Model calculations for band positions composed of two components. (A) Two Gaussian components, (B) linear combinations of the two components, and (C) plot of the position of the linearly combined bands against the fraction of component a. The ratio of displacement between the two bands to full width at half maximum of the bands is 0.25 . The gray line in Panel $C$ represents the relationship of the fraction and the position when the position is linear to the fraction. It should be noted that the position is approximately linearly correlated with the fraction. The same analysis was performed based on the actual absorption spectra of KR2. (D) Absorption spectra of nanodisc-reconstituted KR2 in the presence of $1000 \mathrm{mM} \mathrm{Na}^{+}$(a) and absence of $\mathrm{Na}^{+}(\mathrm{b})$, (E) linear combinations of the two spectra, and (F) plot of the absorption maximum wavelength of the linearly combined bands against the fraction of component a. Again, it should be noted that the absorption maximum wavelength is approximately linearly correlated with the fraction. Based on the linear correlations, we evaluated the cation binding on the basis of the concentration dependence of the absorption maximum wavelength and the Raman frequencies. 

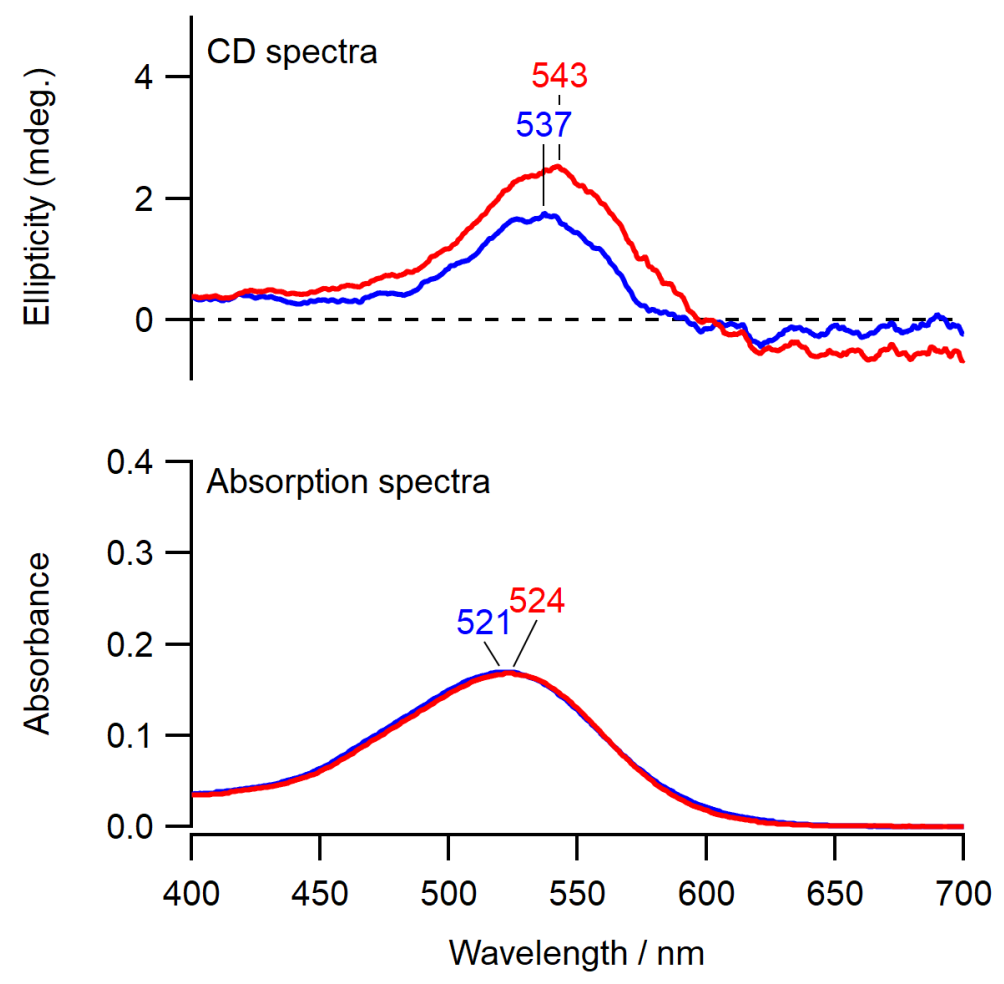

Figure S4. Circular dichroism (top) and absorption (bottom) spectra of nanodisc-reconstituted $\mathrm{KR} 2$ in the presence of $100 \mathrm{mM} \mathrm{Na}^{+}$(red) and choline ion (blue).

Circular dichroism (CD) spectroscopy is commonly used to examine the oligomeric state of microbial rhodopsins. Theoretically, the bilobed spectra with positive and negative peaks is observed due to the exciton coupling between the retinal chromophore in the adjacent protomers when rhodopsins form oligomer. On the other hand, because no exciton coupling occurs in the monomeric rhodopsins, a single positive peak similar to the visible absorption band is observed.

In KR2, a single positive peak was observed in the presence and absence of $\mathrm{Na}^{+}$. The observed CD spectra were in good agreement with that previously reported. ${ }^{1}$ At first glance, these obtained spectra are similar to the characteristics of monomeric rhodopsin. However, the present CD spectra showed that the positive bands were narrower and located at longer wavelength than the band in the absorption spectra (bottom trances). These features in the CD spectra are reasonably explained as the cancelation between the intrinsic positive band and the negative band of the bilobed form due to the exciton coupling, which is expected for the oligomer. If the protein adopts the monomeric structure, it is expected that the bandwidth in CD spectra is similar to that in absorption spectra, which was not observed in the present spectra. Thus, the red-shifted and narrower positive bands indicate that the nanodisc-reconstituted KR2 forms a pentamer both in the presence and absence of $\mathrm{Na}^{+}$. 


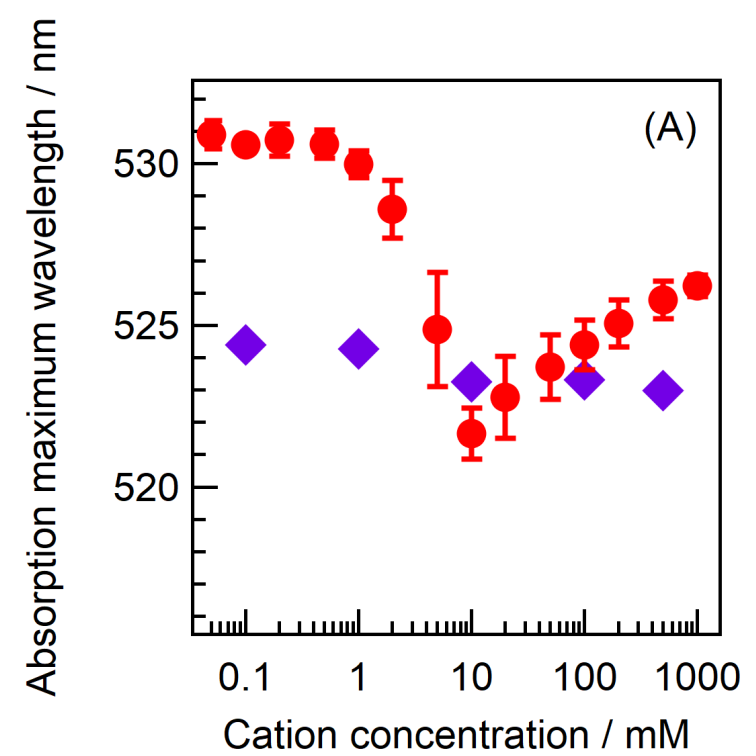

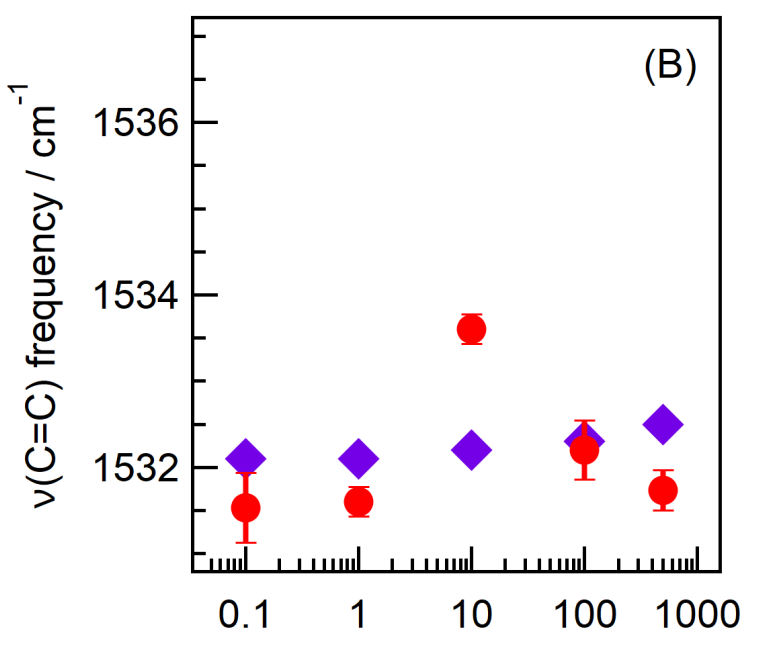

Cation concentration / mM

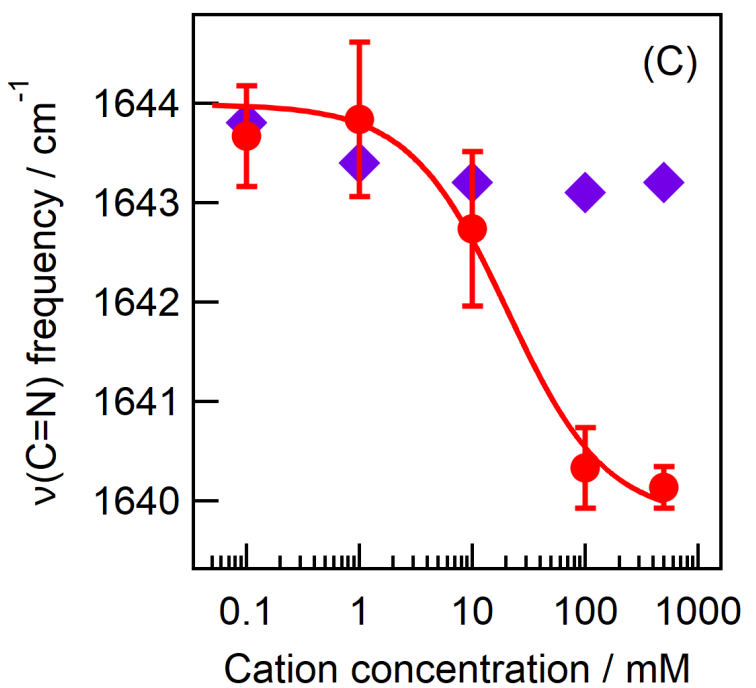

Cation concentration / mM

Figure S5. Sodium-ion concentration dependence of the absorption maximum wavelength (A), $\mathrm{C}=\mathrm{C}$ stretching $(\mathrm{B})$ and $\mathrm{C}=\mathrm{N}$ stretching frequencies $(\mathrm{C})$ of DDM-solubilized KR2. Red circles and purple diamonds show the dependences of wild type KR2 and the D102N mutant, respectively. The red line is the best fit with equation 1 (see text), providing $K_{d}$ value to be $20.6 \pm 7.2 \mathrm{mM}$. 


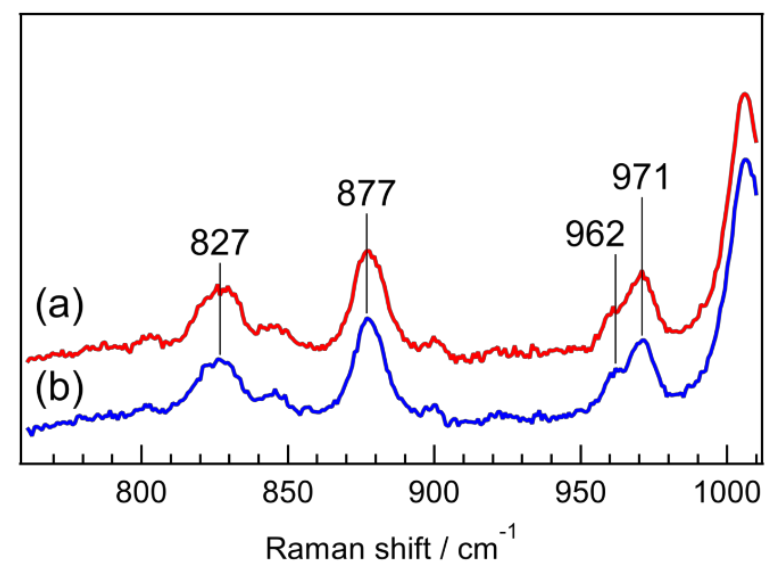

Figure S6. Expanded views of the resonance Raman spectra of KR2 in the region of hydrogen out-of-plane wagging vibrations. Traces $a$ and $b$ show spectra of nanodisc-reconstituted KR2 in buffer containing $100 \mathrm{mM} \mathrm{Na}^{+}$and $\mathrm{K}^{+}$, respectively. 
(A)

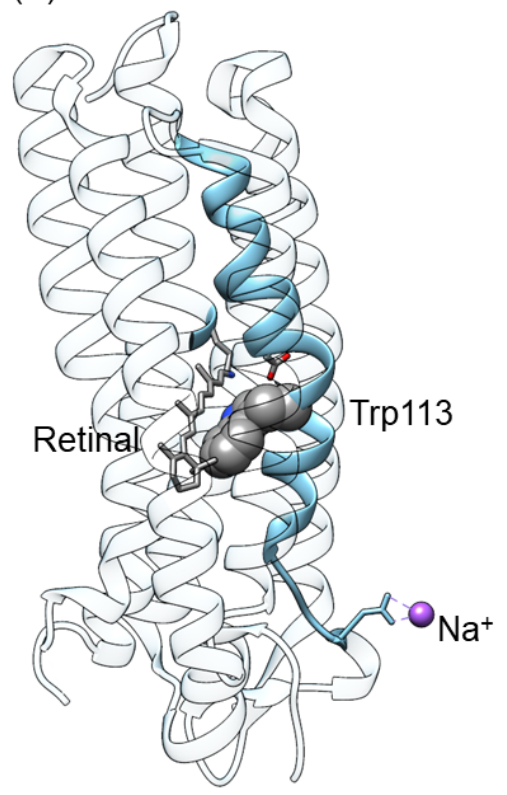

(B)

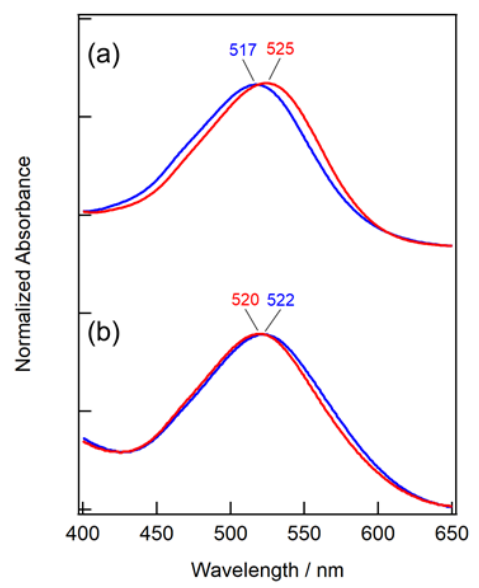

Figure S7. Effect of substitution of Trp113 on the retinal chromophore. (A) Crystal structure of KR2 (PDB: 4XTN). The skyblue helix represents the helix C (Asp102-Leu127). The sphere molecule is Trp113 located in the helix C. (B) The absorption spectra of WT (a) and W113F mutant (b). Red and blue traces show the absorption spectra in the presence of $100 \mathrm{mM} \mathrm{Na}{ }^{+}$and $\mathrm{K}^{+}$, respectively. In $\mathrm{WT}$, the absorption maximum wavelength showed $8 \mathrm{~nm}$ blue shift by replacing $\mathrm{Na}^{+}$with $\mathrm{K}^{+}$. In contrast, W113F mutant showed $2 \mathrm{~nm}$ red shift by replacing them. These results imply that Trp113 contributes to the cation-dependent $\pi$-conjugation changes of the retinal chromophore. 


\section{Reference}

[1] Shibata, M., Inoue, K., Ikeda, K., Konno, M., Singh, M., Kataoka, C., Abe-Yoshizumi, R., Kandori, H., and Uchihashi, T. (2018) Oligomeric states of microbial rhodopsins determined by high-speed atomic force microscopy and circular dichroic spectroscopy, Sci Rep 8, 8262. 